\title{
SIMULATION MODEL FOR DISASTERS AND EMERGENCIES MANAGEMENT FOR SAFETY AND SECURITY IN INDUSTRIAL PLANTS
}

\author{
Francesco Longo \\ University of Calabria \\ Arcavacata di Rende, CS, ITALY \\ E-mail: francesco.longo@unical.it \\ Laura Cirillo \\ University of Calabria \\ Arcavacata di Rende, CS, ITALY \\ E-mail:1.cirillo@msc-les.org \\ Fabio De Felice \\ University of Cassino and Southern Lazio \\ Cassino, FR, ITALY \\ E-mail: defelice@unicas.it \\ Antonella Petrillo \\ University of Naples "Parthenope" \\ Naples, NA, ITALY \\ E-mail: antonella.petrillo@uniparthenope.it
}

\begin{abstract}
The main goal of the work is to carry out research and developments activities to deal with the complexity resulting from emergency management in industrial plants and critical infrastructures.

The project rises from a simultaneous consideration of the two aspects: efficient procedures are needed both on the emergency site and in hospitals; these procedures should be combined with a proper critical patients routing toward the most suitable FirstAid Facilities and with a reduction of human errors during the emergency management. In the process of evaluating emergency alternative problems, there usually exists incomplete and uncertain information, and the decision makers cannot easily express their judgments on the candiates with exact and crisp values. Many MCDM approaches have been proposed to help decision makers to solve problems in uncertain environment. In this paper, the AHP and simulation approach are incorporated to solve group multicriteria decision making problems with incomplete information. The proposed method involves four steps: (1) identify the focal elements of each decision maker according to the emergency scenario; (2) construct the decision model according AHP approach; (3) rank the emergency alternatives; (4) construct the simulation model.
\end{abstract}

Keywords: simulation, emergency management, multicriteria approach. 
Longo, Cirillo, De Felice, Petrillo/ Simulation Model for Disasters and emergencies management for safety and security in industrial plants To Be Submitted to the International Symposium of the Analytic Hierarchy Process 2014, Washington D.C., U.S.A.

\section{Introduction}

The structure of contemporary society greatly affects the detection of industrial disasters. The response to an accident involves the application of a logical and chronological sequence of phases that constitute the chain of rescue and providing relief after the first alarm stage, a continuous collection of data useful for the containment of danger, the sectors of the area of intervention, the critical region, the mobilization and the hospitalization of victims of the disaster. Such operations shall be carried out in order not to saturate the transportation facilities and hospitals, and allow a more fluid evacuation and acceptance process. In this sense it is very important the logistics level of the management and the logic of movement of emergency vehicles by road, or even air, railway, maritime, in cases of particularly tragic case. Organizational effort is to create a harmonious coordination among all entities involved in the intervention by applying to proper management of information flows, integrated communication, monitoring of incoming and outgoing assets from the perimeter. In this context the use of the a multicriteria approach based on AHP (Saaty, 1977) integrated with simulation proves excellent methodological instrument, which allows not only the assessment of the efficiency of managerial choices about the logic of interventions and/or media logistics, but also an accurate identification of organizational problems (Longo et. al, 2012). The development of the model, adopting a very careful approach to the elements of interactivity of the cases under investigation, allows in addition to analyze the behavior of team work in relation to accidents, with the aim of developing training program and develop decision support systems that improve the effectiveness of the intervention.

\section{Literature Review}

A number of natural disasters, such as earthquake, storm surge, Tsunami wave and the flood, etc, occur annually in various parts of the world. In Figure 1 examples of geographical location of disasters are shown.

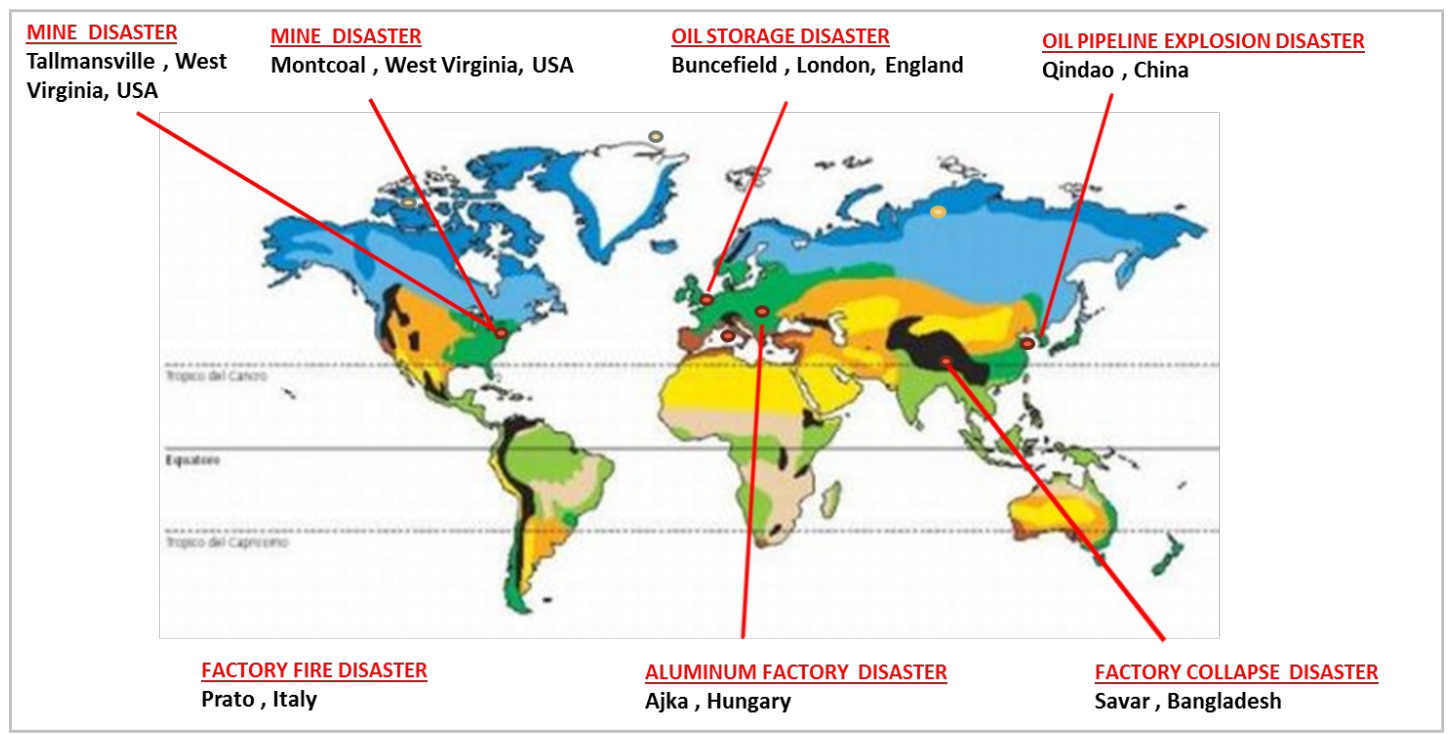

Figure 1: Examples of geographical location of disasters

International Symposium of

Washington, D. C. the Analytic Hierarchy June 29 - July 2, 2014

Process 
Longo, Cirillo, De Felice, Petrillo/ Simulation Model for Disasters and emergencies management for safety and security in industrial plants To Be Submitted to the International Symposium of the Analytic Hierarchy Process 2014, Washington D.C., U.S.A.

In the process of disaster management, a major issue is to evaluate the emergency alternative, and it has attracted much research attention recently (Georgiadoua et al. 2007). As declared by Edrissi et al. (2013) disaster management is a process composed of four phases: namely mitigation, preparedness, response, and recovery. Mitigation is a set of strategic measures taken to reduce, or eliminate the disaster impacts. Preparedness activities are (tactical) measures aimed to lessen or avoid disaster consequences by preparing the community for hazards. Response includes acting according to emergency plans to preserve lives, properties, the environment, and the community 's social, economic, and political structure s. Finally, recovery involves long-term actions that will restore normalcy to the affected areas.

Previous research in management of disastrous events is mainly devoted to studying the four mentioned topics. A key activity in emergency management is planning and preparation for disaster. If the right safety measures are implemented beforehand, harmful effects can be significantly mitigated (Wagner and Agrawal, 2014). For example, a damaged transportation system directly affects the effectiveness of the emergency response plan and has huge socio-economic losses (Okuyama and Chang, 2004). Furthermore in a literature review De la Torre et al. (2012) analyze the potential of operational research models to help relief agencies saves lives, maintain standards of humanitarianism and maximize the use of limited resources.

\section{Hypotheses/Objectives}

The accident scenario definition is a particularly delicate phase that encompasses a multiplicity of potential choices. Key elements considered concern first of all the nature of the business, manufacturing operations carried out inside its territorial dynamic contextualization of failure and its effects, and the presence of structures and technical assistance on health. It was decided to investigate the case of a plant dedicated to synthesizing activity, combustion processes and surface treatment of metals and plastics for the production of tool steel, special pieces, stainless steel. The choice was to contextualize the company geographically in an area of Northern Italy. Nearby, a few miles west, there are major arteries linking Northern Italy. Within the factory there are 54 tons of hydrofluoric acid, 276 tons of hydrofluoric acid at 7\% (toxic), oxygen, $97 \mathrm{t}$ (fuel) 0.07 tons of hydrogen, methane (inflammable) in $2 \mathrm{t}$. The manufacturing process carried on the line consists of a sequence of stages of production such as a material input area, a storage area, furnace and cooling zone, chemical pickling area, storage area, the output area. The triggering of the fire takes place in the first stage of the process, because the rubbing Steel laminated tape against the carpentry of the line. The fire caused the collapse of some pipes containing machining oil, which comes into contact with fire, causes a strong flash fire and a subsequent jet fire affecting the entire working area and invests the workers present.

\section{Research Design/Methodology}

Evaluation and selection of effective measures is difficult due to the numerous scenarios that exist in most emergency environments coupled with the high associated cost of testing such scenarios. The cost of testing multiple scenarios is oftentimes prohibitive (Jain \& McLean, 2008). Thus, evaluation and selection of effective safety measures for 
Longo, Cirillo, De Felice, Petrillo/ Simulation Model for Disasters and emergencies management for safety and security in industrial plants To Be Submitted to the International Symposium of the Analytic Hierarchy Process 2014, Washington D.C., U.S.A.

emergency preparedness is quite difficult and is often left to the subjective judgment of an emergency manager.

First, a problem is defined, including the decision situation and context. Second, objectives are defined, based upon what the different decision-makers and stakeholders value as important. Third, quantitative measures or scales for the objectives are determined. Fourth, alternative choices or strategies are defined. Fifth, the problem is then quantitatively modeled, including probabilistic risk analysis, and the alternatives are ranked in terms of how well they satisfy the objectives.

Sixth, sensitivity analyses are performed in order to examine the impact of uncertainties.

Finally the simulation model is built.

In figure 2 methodological approach is shown.

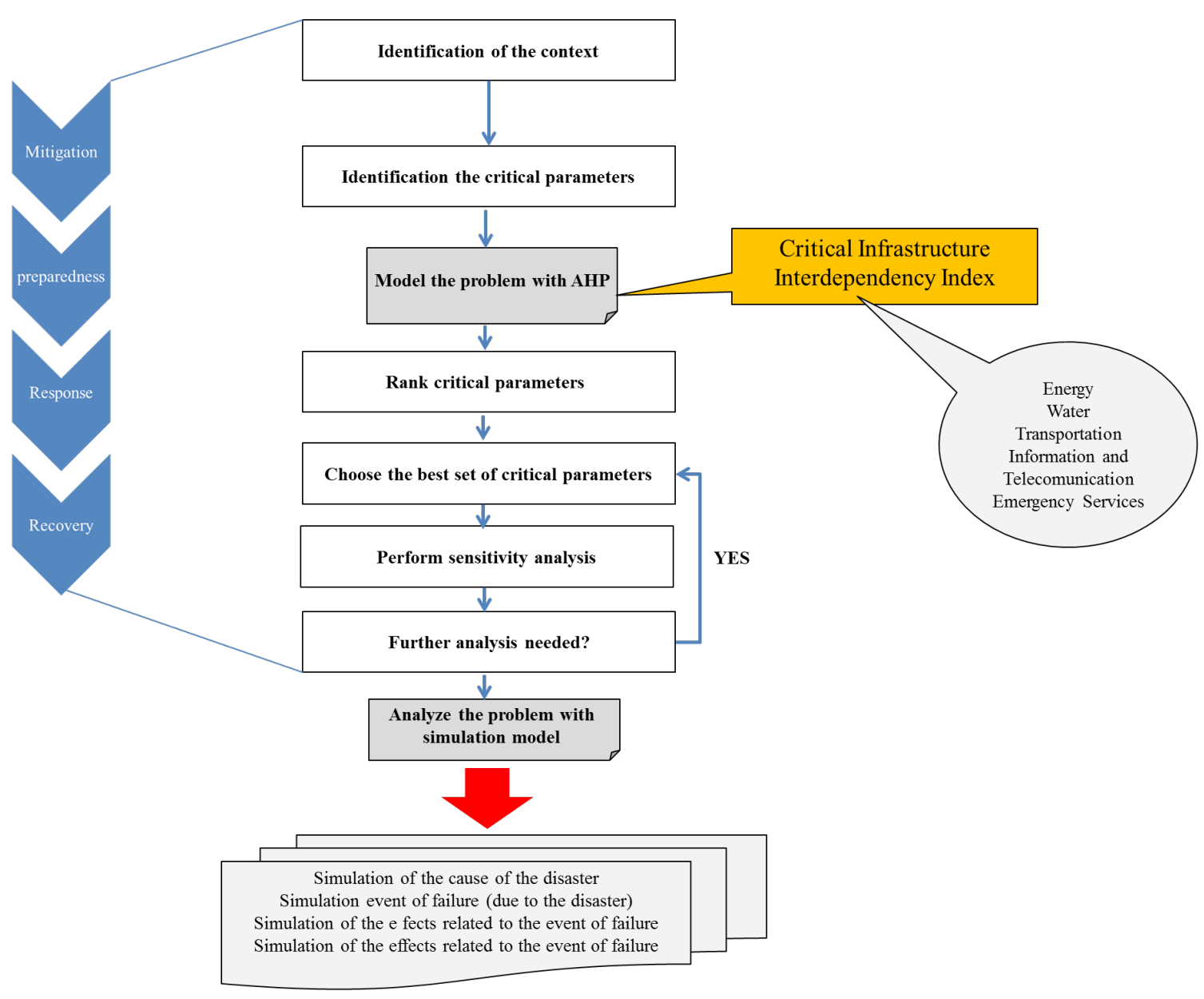

Figure 2: Methodological approach

\section{Data/Model Analysis}

In the present work AHP was used to determine the criteria and elements that best described a disaster and emergency management in a process of prioritization. In figure 3 the AHP model is presented. 
Longo, Cirillo, De Felice, Petrillo/ Simulation Model for Disasters and emergencies management for safety and security in industrial plants To Be Submitted to the International Symposium of the Analytic Hierarchy Process 2014, Washington D.C., U.S.A.

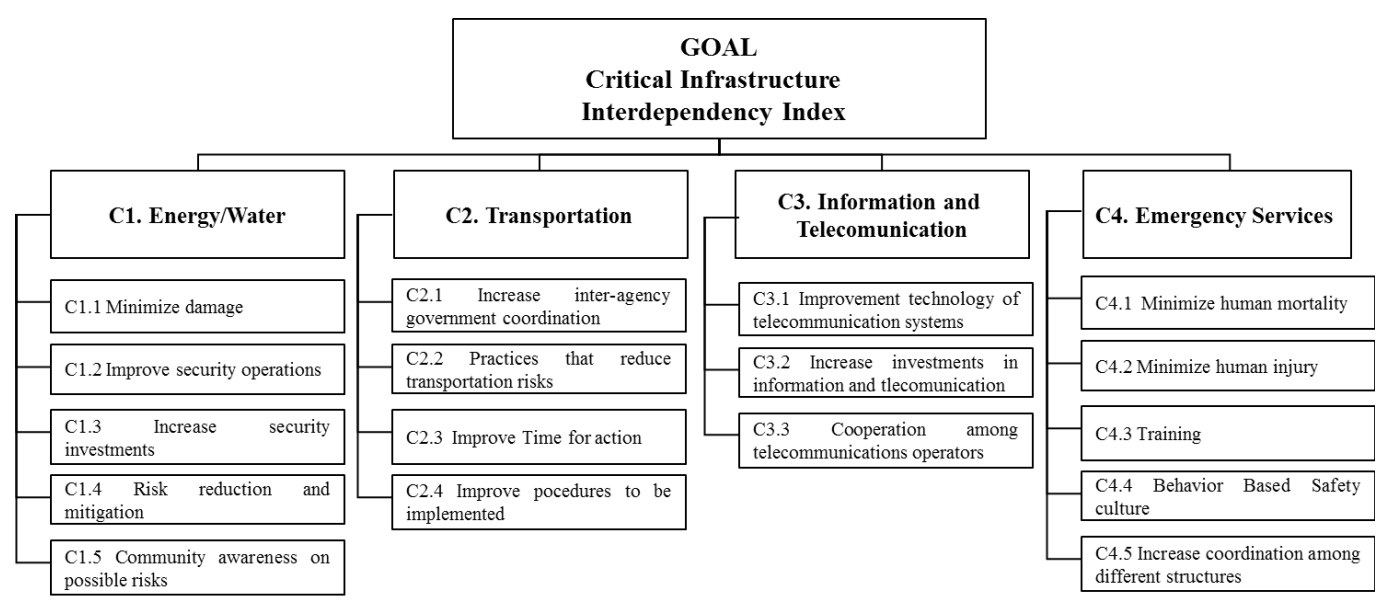

Figure 3: AHP Model

With reference to the AHP model, important alternative criteria and elements associated with achieving a proper disaster and emergency management were identified using paired comparisons and ratio-scale measurement.

An outcome framework was designed based on priority components and were used to determine the outcome indicators of a "index" in order to measure to evaluate the interdependency among critical structures and improve the disaster management.

At present we are working on this model in order to build the simulation model.

\section{Limitations}

The input data necessary for simulation model must be verify in different scenarios. The verification and modification of the model are left for the future work.

\section{Conclusions}

The aim of the paper is to present an integrated approach that combines multi criteria analysis and simulation method for the disaster and emergency management. The use of the integrated model can be a powerful in supporting the disaster management.

\section{Key References}

De la Torre, L.E., Dolinskaya, I.S., Smilowitz, K.R., (2012). Disaster relief routing: integrating research and practice. Socio-Economic Planning Sciences 46 (1), 88-97.

De Felice, F., Petrillo, A. (2011). Methodological Approach for Performing Human Reliability and Error Analysis in Railway Transportation System. International Journal of Engineering and Technology Vol.3 (5), 2011, 341-353.

Edrissi, A., Poorzahedy, H., Nassiri, H., Nourinejad, M. (2013). A multi-agent optimization formulation of earthquake disaster prevention and management. European Journal of Operational Research 229 (2013)261-275 
Longo, Cirillo, De Felice, Petrillo/ Simulation Model for Disasters and emergencies management for safety and security in industrial plants To Be Submitted to the International Symposium of the Analytic Hierarchy Process 2014, Washington D.C., U.S.A.

Georgiadoua, P. S., Papazogloub, I. A., Kiranoudisc, C. T., \& Markatos, N. C. (2007). Modeling emergency evacuation for major hazard industrial sites. Reliability Engineering and System Safety, 92(10), 1388-1402.

Jain, S., \& McLean, C. (2008). Components of an incident management simulation and gaming framework and related developments. Simulation, 84(1), 3-25.

Longo F, Massei M, Nicoletti L (2012). An application of modelling and simulation support industrial plant design. International Journal of modeling simulation and scientific computing, vol. 3, p. 1240001-1-1240001-26, ISSN: 1793-9623.

Okuyama, Y., Chang, S.E., 2004. Modeling Spatial Economic Impacts of Disasters. Springer, 323p.

Saaty, T. L. (1977). A scaling method for priorities in hierarchical structure. Journal of Mathematical Psychology, 15(3), 237-281.

Wagner, N., Agrawal, V. (2014). An agent-based simulation system for concert venue crowd evacuation modeling in the presence of a fire disaster. Expert Systems with Applications 41 (2014) 2807-2815. 